Stam, E., Suddle, K., Hessels, J., y Van Stel, A. J. (2007) "High growth entrepreneurs, public policies and economic growth”. Jena Economic Research Paper, (2007-019), 08-02.

Stevenson, L., y Lundstrom, A. (2002) "Entrepreneurship policy-making: Frameworks, approaches and performance measures". International Council for Small and Medium Sized Enterprises. Washington DC: The World Bank.

Stevenson, L., y Lundström, A. (2007) "Dressing the emperor: the fabric of entrepreneurship policy”. En D. Audretsch, I. Grilo \& A.R. Thurik, eds., Handbook of research on entrepreneurship polic. Northampton, Mass: Edward Elgar Publishing Inc., pp. 94-129.

Studart, R., y Suaznabar, C. (2004) "El acceso al financiamiento, un reto para la creación de empresas dinámicas". En H. Kantis, ed., Desarrollo Emprendedor. America Latina y la experiencia internacional. Washington, D.C. BID-Fundes:111-125.

Tarapuez Chamorro, E., Osorio Ceballos, H., y Botero Villa, J. J. (2013) "Política de emprendimiento en Colombia, 2002-2010”. Estudios gerenciales, 29(128), 274-283.

Verdú, F. M., y Soriano, D. R. (2008) "Rol de la política industrial en el 'entrepreneurship' ¿ cambia la tendencia?”.Información Comercial Española, ICE: Revista de economía, (841), 85-96.

Winchester, L. (2016) Politicas públicas: formulación y evaluación. Santiago de Chile: ILPES/CEPAL.

Winter, S. G. (2006) "Toward a neo-Schumpeterian theory of the firm". Industrial and Corporate Change, 15(1), 125-141.

Zacharakis, A., Shepherd, D. A., y Bygrave, W. D. (2000) Global Entrepreneurship Monitor: National Entrepreneurship Assessment, United States of America: 2000 Executive Report. Kauffman Center for Entrepreneurial Leadership at the Ewing Marion Kauffman Foundation.

Zurbriggen, C., y Lago, M. G. (2014) "Innovación y co-creación. Nuevos desafios para las políticas públicas". Revista de Gestión Pública, 3(2), 329-361.

Cómo citar este artículo

Carballo, Ignacio E., Carlos M. Belloni, Martín López Amorós y Eduardo

L. Fracchia (2017) "Emprendedorismo y políticas públicas. Una introducción a la

literatura". Revista Perspectivas de Políticas Públicas vol. 7 No 13.

\title{
El proceso de construcción de las necesidades en las políticas habitacionales. Un modelo teórico - metodológico para su análisis desde una perspectiva interpretativa
}

Shaping housing needs in public policies. A theoretical - methodological model from an interpretive perspective.

Venettia Romagnoli Arquitecta, Magister en Gestión del Ambiente, el Paisaje y el Patrimonio Docente de la Facultad de ArqLACSO y Urbanismo de la Universidad Nacional del Nordeste (FAU- UNNE). Investigadora Adiunta UNNE-CONICE. Investigadora Adjunta UNNE-CON

Fecha de recepción: 28.4.17

Fecha de aceptación 6.8.17

\section{Resumen}

El trabajo que aquí se presenta fue desarrollado en el marco de la tesis doctoral recientemente finalizada, denominada "¿Qué vivienda necesitan los pobres? Un estudio sobre la configuración de las necesidades en las políticas habitacionales vigentes a partir del caso del AMGR (Chaco, Argentina)", que se dedicó a analizar el proceso de configuración de las necesidades habitacionales en las políticas públicas, para identificar sus diferentes momentos, los actores involucrados, sus intereses, roles, estrategias e incidencia en la politización, interpretación e implementación de dichas necesidades. Este artículo se dedica al desarrollo del modelo teórico - metodológico construido para el análisis del fenómeno mencionado. 
Palabras clave: necesidades humanas - políticas habitacionales - modelo teórico - metodológico de análisis - Política Federal de Vivienda Argentina.

\section{Abstract}

This paper was produced within the framework of the recently completed doctoral thesis, called "What housing do the poor need?. A study on the configuration of needs in housing policies in force since the case of the AMGR (Chaco, Argentina) ", was devoted to analyze the process of shaping housing needs in public policies, to identify their different moments, actors involved, their interests, roles, strategies and impact on the politicization, interpretation and implementation of those needs. This article is devoted to the development of a theoretical-methodological model built for the analysis of this process.

Key-words: human needs - housing policies - theoreticalmethodological model - Argentina's Federal Housing Policy.

\section{Introducción}

El presente trabajo forma parte del desarrollo de una tesis doctoral dedicada al análisis del proceso de configuración de las necesidades habitacionales en las políticas públicas respectivas. Para analizar dicho proceso resultó necesario avanzar en la construcción de un marco interpretativo propio y un modelo teórico- metodológico, cuya explicitación y desarrollo es el objetivo principal de este artículo. La hipótesis orientativa de la investigación estuvo orientada a destacar que las políticas públicas son construidas en un proceso de disputa entre diversos actores asimétricos que ponen en juego estrategias y recursos con el fin de incidir en las definiciones que terminan siendo legitimadas por el Estado precisamente a través de las políticas públicas y sus instrumentos operativos: los programas y los proyectos.

Entender que las necesidades que originan y fundamentan una determinada política habitacional no están simplemente dadas ni son construidas técnicamente de una manera aséptica por un determinado gobierno, sino que son el resultado de un proceso de configuración social, plantea la necesidad de avanzar en la construcción de un marco interpretativo propio del concepto de necesidades humanas, basado en los aportes teóricos desarrollados por autores que las visualizan como resultantes de procesos de configuración atravesados por la disputa entre diversos actores. Desde diferentes roles y en función de intereses esos actores despliegan estrategias con el fin de incidir en las interpretaciones que terminan legitimándose a través de la intervención del Estado (Fraser 1991; Krmpotik 1999). Este marco interpretativo propio se nutrió también de conceptualizaciones de las políticas públicas que, superando los enfoques tecnocráticos, las interpretan como producto de un espacio de disputa entre actores (Subirats 1989; Tamayo Saenz 1997; Jobert 2005; Bourdieu 1993, 1998 y 2001, entre otros) entendiendo que las mismas son definidas por un marco normativo y sus instrumentos operativos: los programas y los proyectos (Romagnoli 2010, 2011; Barreto 2010; Barreto y otros 2010 y 2014). El ensamblaje de estas dos vertientes de estudios teóricos permitió conformar un esquema conceptual crítico, a partir del cual se diseñó un modelo teórico-metodológico para analizar el mencionado proceso.

Con base en lo expuesto, se desarrollan en primer lugar los principales fundamentos metodológicos que se han derivado del marco interpretativo construido, para avanzar posteriormente en el planteo del modelo teórico- metodológico propuesto para el estudio del proceso de configuración de las necesidades habitacionales y la definición de los observables de investigación. Seguidamente, en función de ejemplificar la utilidad analítica del mencionado modelo, se presenta la aplicación del mismo a un caso de estudio, para finalizar con un conjunto de reflexiones relativas a su capacidad analítica para el estudio de las políticas habitacionales desde una perspectiva interpretativa.

\section{Fundamentos metodológicos}

El tema de las necesidades humanas ha sido ampliamente discutido en el campo de las ciencias sociales Maslow, 1954; Heller 1986 y 1996; Max Neef, Elizalde y Hopenhayn 1986; Doyal y Gough 1991 y 1994; Gough 2003 y 2008; Sen 1987, 1992, 1997 y 2000; Nussbaum 1992 y 2002, Gasper, 2002; Ibrahim 2006, por mencionar algunos de los grandes referentes de diferentes posiciones sobre el tema).

En el campo del hábitat social y de las políticas habitacionales, las necesidades se han tomado como "simplemente dadas", circunscribiéndose las discusiones en torno a los satisfactores de las mismas. En las escasas investigaciones en las que se ha avanzado sobre el tema ha prevalecido una visión sectorial, señalándose que las inadecuaciones de las soluciones brindadas por la política habitacional radican en el hecho de que en la gestión pública convencional de la vivienda, las necesidades son definidas a partir de conjeturas o hipótesis más o menos racionalmente fundadas, por su traducción como carencia de determinados satisfactores estandarizados o, en el mejor de los casos, por perfiles abstractos de necesidad basados en aproximaciones estadísticas (Pelli, 2006). Las limitaciones de estas conceptualizaciones se han adjudicado al carácter técnico de las 
interpretaciones o a la falta de incorporación de la voz de los beneficiarios, invisibilizando el proceso político de disputa en el cual se construyen las necesidades (Fraser 1990).

Con el fin de llevar a cabo un abordaje de mayor complejidad, se planteó como hipótesis orientativa de la investigación que dio origen a la estrategia y al modelo de análisis que se explicitan a continuación, que las necesidades que fundamentan la implementación de políticas públicas son construidas en un proceso de disputa entre actores asimétricos que ponen en juego estrategias y recursos con el fin de incidir en las definiciones que son legitimadas por el Estado a través de las políticas públicas y sus instrumentos operativos (los programas y proyectos).

El método general con el que se llevó a cabo la investigación consistió en un modelo inductivo-cualitativo. Al no encuadrarse en una teoría sustantiva, se recurrió a una estrategia fuertemente inductiva, que requirió del desarrollo de un marco interpretativo propio y de un modelo teórico metodológico con el que analizar el objeto general de estudio: el proceso de configuración de las necesidades en las políticas habitacionales.

Del estudio teórico y de la construcción del marco interpretativo propio, surgieron los fundamentos metodológicos, entre los que destacan:

- Entender a la política habitacional como el resultado de un proceso de configuración de las necesidades habitacionales, implicó la posibilidad de distinguir sus diferentes momentos e instancias y relacionarlos con los elementos constitutivos de la política: su marco normativo general y sus instrumentos operativos: los programas y los proyectos, que representan a su vez sus distintos niveles.

- Las políticas habitacionales, de acuerdo al modelo de desarrollo y los lineamientos que propicien pueden ser "enlatadas", dejando pocos espacios de discusión para su definición en los niveles jurisdiccionales inferiores, o pueden ser más "flexibles", permitiendo la introducción de mayores definiciones en las instancias de los programas y los proyectos. Cuestión clave al vincular la política con el proceso de configuración de las necesidades habitacionales desde una perspectiva interpretativa, en la cual cobran importancia analítica los actores que participan de dicho proceso, ya que son estos agentes quienes, desde los diferentes niveles jurisdiccionales involucrados y desde diversos roles, despliegan estrategias y ponen a jugar los recursos con los que cuentan para incidir tanto en la politización de una determinada necesidad, como en su interpretación y traducción a un nuevo servicio social y su posterior implementación.

- En el proceso de configuración de las necesidades polemizan diferentes tipos de actores, que pueden distinguirse en función de los tipos de discursos de los que se constituyan como portadores. En primer lugar, identifica los discursos que tienen como meta politizar necesidades previamente privatizadas y que son iniciados por personas subordinadas o grupos involucrados en el diseño de nueva identidades sociales de oposición. En segundo lugar reconoce la existencia de discursos cuya meta es reprivatizar necesidades recientemente politizadas y defender la división social establecida entre discursos al articular interpretaciones enraizadas en necesidades que podían darse por dadas anteriormente, y que generalmente corresponden grupos hegemónicos. Finalmente, también entran en juego los discursos que traducen necesidades politizadas a necesidades administrables, incluyendo tanto discursos terapéuticos de trabajadores sociales profesionales y discursos de sistemas funcionalistas de planificadores, expertos en políticas y científicos sociales de la corriente dominante.

\section{Esquema teórico - metodológico}

Los fundamentos teóricos planteados precedentemente fueron plasmados en el siguiente esquema que permite visualizar los diferentes momentos del proceso de configuración de las necesidades, los actores que inciden en el mismo y los niveles de la política implicados. 
Gráfico 1. Relación teórica - metodológica entre los momentos del proceso de configuración de las necesidades y los niveles de la política.

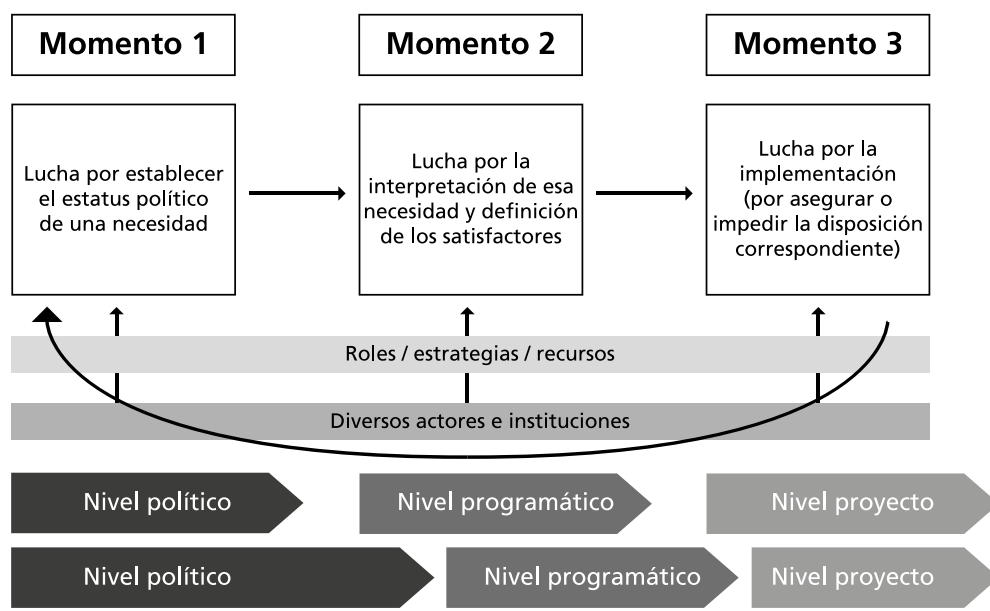

Fuente: Romagnoli (2015)

El esquema analítico construido permite inferir la existencia de tres momentos fundamentales en el proceso de configuración de las necesidades habitacionales en las políticas públicas. El primer momento corresponde, tal como ha sido señalado, a la disputa por lograr o impedir la implantación del estatuto político de la necesidad habitacional, la lucha para validar la necesidad como una cuestión de legítima preocupación pública o para enclavarla como una cuestión privada. Usualmente, una condición para que una cuestión sea pública en el sentido del Estado es que primero sea pública en el sentido del discurso; lo que quiere decir que una necesidad no se vuelve sujeta a la intervención estatal legítima sino hasta que ha sido sujeta al discurso o argumentos colectivos.

Esta instancia implica la politización de ciertas necesidades específicas a través de determinados procesos, mediante los cuales algunas necesidades "latentes" rompen las envolturas de lo privado que previamente las contenían y se vuelven focos de discurso público contestatario. En términos de Fraser (1986) las necesidades se politizan cuando ciertos grupos comienzan a cuestionar sus identidades y posiciones subordinadas y las interpretaciones acerca de sus necesidades tradicionales y desventajosas, siendo los principales actores de esta instancia los movimientos sociales de oposición y el Estado.

Las estrategias que despliegan los actores para lograr legitimar sus necesidades requieren además de un contexto propicio para su surgimiento, configurado por determinadas circunstancias y por la incidencia de ciertos factores económicos y culturales que en un momento dado favorecen la desorganización o interrupción de los procesos de despolitización que habían mantenido a dichas necesidades cautivas en enclaves privatizadores tales como "lo doméstico" o "lo económico", volviéndose permeables al surgimiento de interpretaciones opuestas que habían permanecido subordinadas (Fraser 1986, 1991).

Finalmente, como cierre provisional de la caracterización teórica de este primer momento, cabe señalar que la politización de necesidades no se da sin oposición. En este escenario están presentes también los grupos que intentarán reprivatizar estas necesidades. Estas dos formas de discurso polemizan en un espacio de disputa, y al hacerlo modifican sutilmente sus interpretaciones de las necesidades.

El segundo momento del proceso contempla la lucha por la interpretación de la necesidad, la disputa por definirla y así determinar qué podría satisfacerla; es un momento que tiene lugar cuando las necesidades cuestionadas han logrado establecer su status público y político. Aquí el punto focal ya no es lo "público" frente a lo "privado" en el sentido del discurso, sino más bien el contenido y el proceso de la interpretación de la necesidad. Las interpretaciones en competencia, desarrolladas por los movimientos sociales, tienden a traducirse en demandas de satisfacciones organizadas políticamente, plasmándose en concepciones programáticas opuestas.

En esta instancia, el Estado se constituye en un actor protagónico en la lucha por las interpretaciones conflictivas de necesidades dentro del espacio de "lo social". A través de sus agencias a nivel federal, provincial y local, el Estado se hace cargo de regular y fundar o satisfacer necesidades sociales antes privatizadas, a través de "servicios sociales", cuyo diseño e instrumentación se ocupa, igualmente de interpretar como así de satisfacer necesidades sociales (Fraser 1986,1991$)$

El encuentro entre las necesidades exitosamente politizadas con el Estado implica una serie de operaciones de re-escritura, un conjunto de procedimientos mediante los cuales las necesidades exitosamente politizadas se traducen a necesidades administrables. Para Fraser (1986), el programa Estado para reescribir las necesidades politizadas es el siguiente: la necesidad politizada se redefine como un concepto correlativo de una satisfacción burocráticamente administrable, un servicio social, lo que requiere de criterios claros y objetivos de elegibilidad tales que una agencia organizada burocráticamente pueda determina quién tiene la necesidad y quién no. Esto, a su vez, significa que la necesidad debe especificarse en términos de un estado de cosas generalizable que podría aplicarse en principio a cualquiera (ej. desempleo, discapacidad, etc.), en consecuencia, dicha necesidad debe ser descontextualizada y despojada de los significados sociales e individuales que adquirió en el pasaje de la privatización a la politización. Como resultado de este proceso, se reubica al poseedor de la necesidad y el Estado lo interpela de una manera simultáneamente individualizadora y generalizadora. El poseedor de la necesidad se reubica como un individuo demandante que debe demostrar que su caso satisface los criterios de elegibilidad, relegando su posición como agente político o miembro de una colectividad constituida. 
Esto implica que el problema politizado de la vida del demandante debe concordar con una solución administrable burocráticamente y volverse conmensurable en términos de tal solución. Esto quiere decir que la necesidad debe cuantificarse y redefinirse como el equivalente en una cantidad de dinero (recursos), operación que sin dudas descontextualiza y reubica al poseedor de la necesidad. En estos procesos, los sujetos portadores de las necesidades son interpelados como receptores pasivos de servicios predefinidos, no como participes involucrados en la interpretación de las necesidades y conformación de sus propias condiciones de vida.

Además de estas operaciones descontextualizadoras, individualizadoras, generalizadoras y apaciguadoras, se entiende que la definición administrativa de las necesidades incluye también una fuerte operación normalizadora. A medida que las necesidades y sus poseedores se vuelven conmensurables unas con otros, tienden a ser colocados en escalas de normalidad y desviación relativas construidas estadísticamente. Como resultado, el diseño de los servicios tiende a adoptar el carácter de normalización.

Para Fraser (1986) está claro que la manera que el Estado tiene de interpretar las necesidades y de interpelar a los sujetos se contrapone con las prácticas que los movimientos sociales habían desarrollado para politizar sus necesidades. En este segundo momento, la lógica administrativa del Estado tiende a despolitizar estas necesidades, sin repatriarlas a sus enclaves privatizadores anteriores, sino más bien reformulándolas dentro de una envoltura administrativa, en donde los administradores y profesionales definen las necesidades de sus "clientes" con antelación y los sujetos son individualizados como "casos" en formas que militan contra la identificación colectiva y la construcción de capital social, en términos de Bourdieu $(1985,1993)$.

Sin embargo, no todo el escenario es negativo desde una perspectiva que promueve la visibilización y democratización del proceso de configuración de las necesidades en las políticas públicas, como la que desde esta investigación se sostiene. En algunos casos, bajo ciertas condiciones, Fraser (1986) advierte que los clientes de la seguridad social logran desplegar estrategias de resistencia a la lógica estatal, desplazando el significado y carácter de la interpelación que se les impone, pueden transformar la posición de cliente con un estigma que denota dependencia, atomización y debilitamiento, en una identidad política, la identidad política de una colectividad constituida que denota activismo, solidaridad y adquisición de poder.Al hacerlo, tales clientes cuestionan simultáneamente las definiciones administrativas de sus necesidades y la lógica anti participativa y jerárquica de la definición administrativa de los servicios sociales que les impone el Estado.

En términos analíticos, se considera que las interpretaciones y definiciones que logran imponerse como resultado de la disputa que se da en esta instancia, quedan plasmadas en la letra escrita de los instrumentos operativos de la política (los programas y los proyectos), constituyéndose a partir de esta institucionalización, en la manera legitimada por el Estado de entender determinado problema y su abordaje.
Finalmente, se entiende que en el tercer momento del proceso de construcción de las necesidades habitacionales se materializa la lucha por la traducción de la interpretación de necesidad habitacional y la definición de los satisfactores plasmada en la letra escrita de los instrumentos operativos al lenguaje de la intervención. Aquí la disputa ya no está centrada en la interpretación, sino concretamente en la satisfacción efectiva de la necesidad, es una lucha por asegurar o impedir la disposición correspondiente.

Esta última instancia corresponde a la implementación, entendiendo que en este momento del proceso se materializan una sucesión de acciones de diversos actores asimétricos que desde diferentes roles, despliegan estrategias en virtud de incidir en las decisiones en relación a las condiciones en las cuales se entregarán los satisfactores que componen el programa y el proyecto, definidos en los momentos previos del proceso de configuración de las necesidades. Esta concepción implica que el problema de la implementación no es el ajuste fiel a la formulación de los instrumentos operativos, sino su adaptación a las características locales, al resultado de las disputas de los actores para monopolizar e incidir en la traducción de la necesidad administrable a la ejecución concreta. La implantación, en definitiva, es un proceso, no un momento y además, un proceso dificilmente distinguible de la decisión, puesto que en él también se adoptan una multitud de decisiones (Tamayo Sáez 1997: 13-17).

En relación a los diferentes tipos de actores que participan del proceso, se planteó la necesidad de distinguirlos y tipificarlos en función de los tipos de discursos de los cuales se constituyan como portadores, tal como se esquematiza en el Gráfico 2.

Gráfico 2. Esquema de actores y discursos en disputa según Fraser (1986 y 1999).

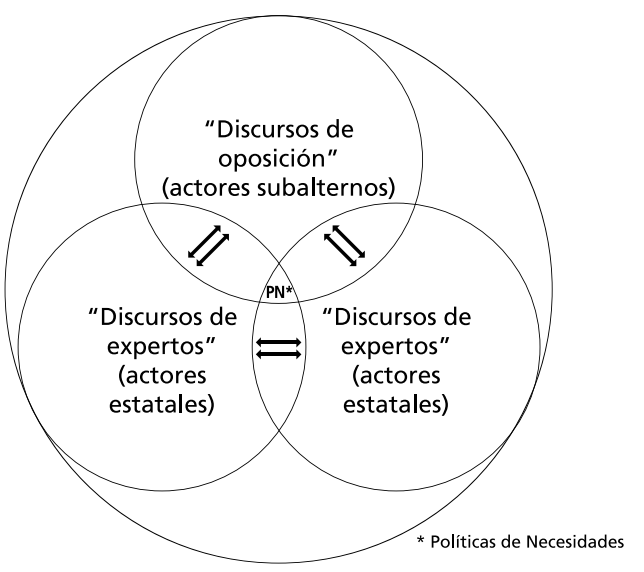

Fuente: Romagnoli (2015) 
A partir de la identificación y clarificación teórica y metodológica de los distintos momentos del proceso de configuración de las necesidades habitacionales en las políticas públicas, se consideró que su aplicación como matriz de análisis puede contribuir a echar luz sobre algunas características importantes del proceso que sustenta la política habitacional, ilustrando además ciertas dimensiones de la interacción de los distintos tipo de actores involucrados.

\section{Construcción teórico - metodológica de los observables de investigación:}

En base a lo expuesto hasta aquí, debe señalarse que desde el punto de vista metodológico, se entendió que el proceso de configuración de las necesidades habitacionales en las políticas vigentes puede reconstruirse a partir del análisis de los discursos (escritos $\mathrm{y}$ orales) de los diversos actores que participaron e incidieron de manera indirecta $\mathrm{y} / \mathrm{o}$ directa con mayores y/o menores recursos de poder en la legitimación de una determinada visión acerca de las necesidades habitacionales, los satisfactores “adecuados” y el tipo de respuesta que terminó efectivamente brindando el Estado.

Desde este enfoque, se consideró que los documentos de la política habitacional y de sus instrumentos operativos (marcos normativos, leyes, decretos, planes, resoluciones, manuales operativos, etc.), que se constituyen en los discursos escritos mencionados, representan un insumo significativo para analizar los "supuestos teóricos" que fundamentan las intervenciones que se producen en el marco de una determinada acción política. En este sentido, se entendió por "supuestos" a las teorías no explícitas en la letra de un programa pero en las que se fundan la validez de sus procedimientos. Los mismos se desprenden de analizar la letra de los programas en relación con los conceptos que sustentan su contenido, proveniente de elaboraciones académicas. Finalmente, se concibió que el cuerpo de teorías que se encuentran detrás de los programas vigentes en un período define la orientación general de la política habitacional, y el análisis de cada programa en particular permite encuadrarlo dentro de la orientación así definida.

Si bien se consideró que las fuentes documentales mencionadas pueden constituirse en insumos fundamentales para el análisis de las interpretaciones de las necesidades habitacionales que fundamentan determinados tipos de intervenciones (estén estas planteadas de manera explícita o no en los documentos oficiales), las mismas no son suficientes para dar cuenta de las disputas implicada en el proceso de configuración de las necesidades, sino que deben ser complementadas con los discursos orales de los actores que han participado de dicho proceso, y que permitirán reconstruir los mecanismos y recursos de poder utilizados por ellos, que representaron intereses en muchos casos contrapuestos y que lucharon por incidir y posicionar su concepción a partir de la legitimación que otorga el poder estatal.

\section{La aplicación del modelo teórico - metodológico a un estudio de caso}

Resulta necesario en este punto precisar algunas cuestiones referidas al objeto de estudio específico que fue analizado a la luz del marco interpretativo, la estrategia y el modelo teórico - metodológico, con el objeto de precisar y ejemplificar cómo el modelo permitió avanzar en la definición operativa de los objetos de análisis, las unidades de análisis, los observables específicos y las técnicas y procedimiento de relevamiento y análisis de los datos inclusive.

La investigación a partir de la cual fue diseñada la metodología y el modelo teórico-metodológico tuvo como objeto el proceso de configuración de las necesidades en la Política Federal deVivienda vigente en Argentina en el período 2003- 2007, a partir del análisis en profundidad de tres proyectos implementados en el Área Metropolitana Gran Resistencia (Provincia de Chaco), haciendo un esfuerzo por reconstruir sus diferentes momentos, desentrañar sus particularidades, los actores involucrados, sus roles, estrategias e incidencia en la politización, interpretación/definición e implementación de las necesidades habitacionales, que determinaron las definiciones finalmente legitimadas por el Estado y ejecutas en el territorio.

Si bien de acuerdo al esquema teórico -metodológico construido, se entendió que es posible identificar y relacionar con fines analíticos los distintos momentos del proceso de configuración de las necesidades habitacionales (la disputa por el reconocimiento, la disputa por la interpretación y la disputa por asegurar o impedir la disposición correspondiente) con los diferentes niveles de la política habitacional (política general, programas y proyectos), esta investigación definió a los proyectos como objeto empírico principal, en función de las limitaciones y posibilidades concretas de acceso a las fuentes de información, considerando que el enfoque metodológico adoptado situaba en un mismo nivel de jerarquía como fuentes de información para el análisis a los discursos escritos (documentos de la política habitacional y de sus instrumentos operativos: marcos normativos, leyes, decretos, planes, resoluciones, manuales operativos, etc.) y a los discursos orales de los actores involucrados en el proceso que se requería indagar (funcionarios, técnicos, destinatarios, académicos, empresarios, etc.).

Los niveles superiores de la política -la política habitacional general (Plan Federal de Vivienda) y sus programas orientados a los sectores en situación de pobreza (Programa Federal de Emergencia Habitacional, Programa Federal de Mejoramiento de Viviendas y Programa Federal de Solidaridad Habitacional)- fueron analizados principalmente a partir del estudio de su letra escrita y de resultados de investigaciones anteriores, con el fin de reconstruir el proceso de configuración de las necesidades habitacionales subyacente a los instrumentos operativos. Para el estudio, se tuvo en cuenta la gestión gubernamental comprendida entre mayo 2003 y diciembre 2007 (presidencia de Néstor Carlos Kirchner), período que representó un cambio en la política habitacional anterior. El límite temporal de finales de 2007 obedeció a la conveniencia de contar con una distancia suficiente de análisis de los hechos ocurridos. 
A partir del propósito de la investigación y de diferenciar estos niveles y escalas de procesos descriptos, resultó necesario avanzar en la definición operativa de los objetos de análisis. Para poder identificarlos se interpeló al modelo teórico-metodológico construido, que distingue diferentes momentos del proceso de configuración de las necesidades habitacionales de la PFV, haciendo un esfuerzo por explicitar las preguntas iniciales que orientaron su análisis. En el siguiente gráfico se esquematiza el proceso metodológico señalado.

Gráfico 3. Esquema síntesis de la estrategia metodológica en función del enfoque teórico
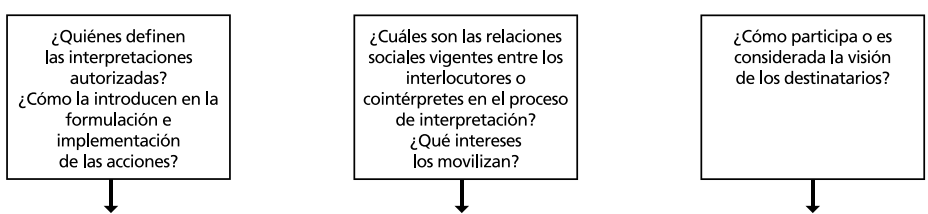

\section{Momento 1}

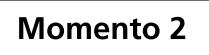

Momento 3
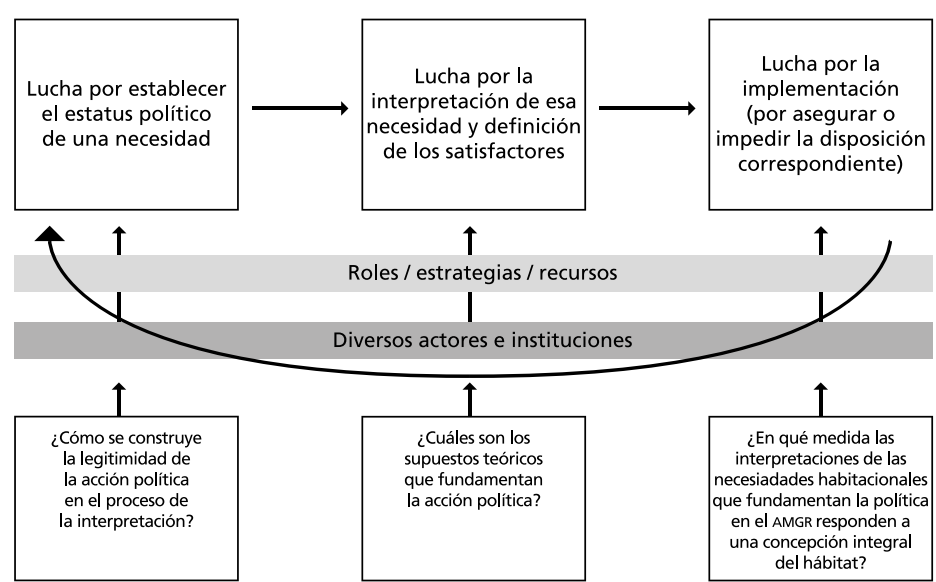

Fuente: Romagnoli (2015)

A partir del esquema precedente se definieron como unidades de análisis a los tres momentos identificados en el modelo teórico-metodológico con el objetivo de reconstruir, a luz de los mismos, el proceso de configuración de las necesidades habitacionales en las políticas vigentes a partir su implementación en el Área Metropolitana Gran Resistencia (AMGR). En cada uno de los momentos se propuso desentrañar lo acontecido en cada instancia, identificando los actores involucrados, sus roles, estrategias e incidencia en la politización, interpretación/definición e implementación de las necesidades habitacionales.
Gráfico 4. Estructura del análisis

\begin{tabular}{|l|l|c|c|}
\hline \multirow{2}{*}{ MOMENTOS CASOS } & \multicolumn{3}{|c|}{$\begin{array}{c}\text { CASOS } \\
\text { (Proyectos ejecutados en el AMGR en el marco } \\
\text { de programas de la PFV) }\end{array}$} \\
\hline & \multicolumn{1}{|c|}{ Caso 1 } & Caso 2 & Caso 3 \\
\hline $\begin{array}{l}\text { Momento 1 } \\
\text { Lucha por establecer el status } \\
\text { político de la necesidad }\end{array}$ & $\begin{array}{l}\text { Roles, etrategias } \\
\text { e incidencia de los } \\
\text { actores }\end{array}$ & & \\
\hline $\begin{array}{l}\text { Momento 2 } \\
\text { Lucha por la interpretación } \\
\text { de la necesidad y definición } \\
\text { de los satisfactores }\end{array}$ & $\begin{array}{l}\text { Roles, etrategias } \\
\text { e incidencia de los } \\
\text { actores }\end{array}$ & & \\
\hline $\begin{array}{l}\text { Momento 3 } \\
\text { Lucha por la implementación }\end{array}$ & $\begin{array}{l}\text { Roles, etrategias } \\
\text { e incidencia de los } \\
\text { actores }\end{array}$ & & \\
\hline
\end{tabular}

Fuente: Romagnoli (2015)

Finalmente, en el Gráfico 5 se detallan -a partir del modelo teórico - metodológico construido- los objetos de análisis en cada uno de los niveles (político, programático y de proyectos) de la política habitacional, como así las técnicas de relevamiento de los datos que permitieron reconstruir los momentos del proceso de configuración de las necesidades habitacionales. 
Gráfico 5. Esquema de la estrategia metodológica con definición de los objetos de análisis, observables específicos, casos y técnicas utilizadas

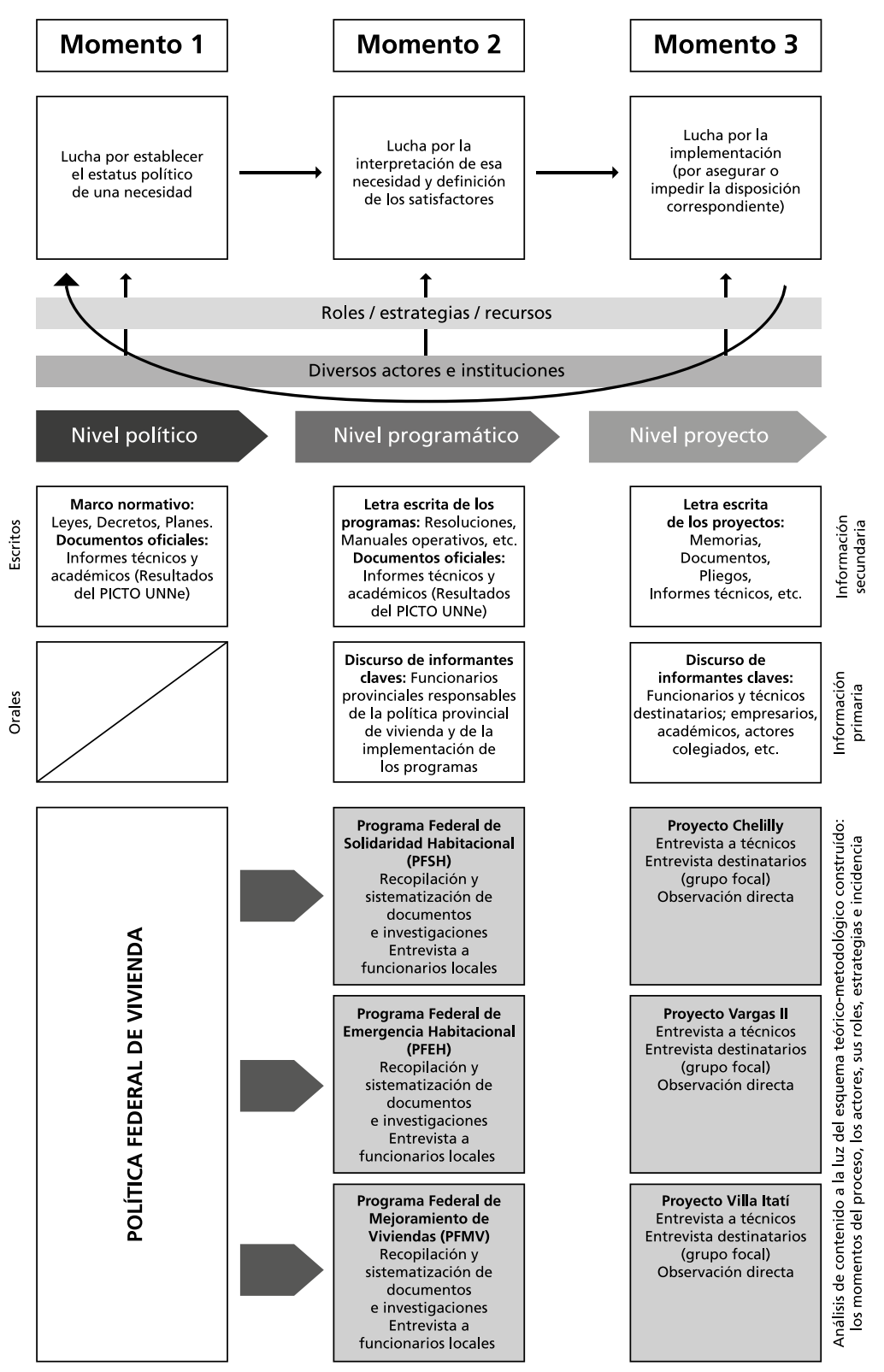

Fuente: Romagnoli (2015)

\section{Consideraciones finales}

La investigación resumida en las secciones precedentes permitió confirmar la hipótesis orientativa que señalaba que las necesidades habitacionales en las políticas públicas no estaban simplemente dadas ni eran formuladas de manera técnica por el Estado. Por el contrario, tal como pudo verificarse, a partir de la estrategia metodológica y su aplicación a la implementación de la Política Federal deVivienda en el AMGR (2003-2007), las necesidades habitacionales se configuran en un proceso de disputa entre actores asimétricos que despliegan estrategias y asumen diferentes roles en cada uno de los momentos que atraviesa dicho proceso, en virtud de incidir en el reconocimiento, la interpretación y la implementación de dichas necesidades.

Con base en lo señalado, se entiende que más allá de los hallazgos propios de la investigación, el enfoque teórico metodológico expuesto en este artículo constituye en sí mismo en un producto que puede aportar importantes instrumentos analíticos para la construcción de un marco interpretativo crítico de análisis de la politica habitacional.

La potencialidad de los aportes teóricos y metodológicos resultantes de la investigación al campo de los estudios de la política habitacional, se sustenta desde un posicionamiento que entiende que la visibilización del proceso de construcción de las necesidades en las políticas públicas, puede constituirse en un primer aporte hacia el logro de una mayor democratización del mismo, lo que requerirá sin dudas de un fuerte protagonismo del Estado, promoviendo la participación de diversos actores políticos, comunitarios, económicos, académicos, mitigando las imposiciones que algunos sectores puedan ir logrando sobre otros, en pos de lograr una distribución equitativa de la participación para el establecimiento de consensos, integrando la dimensión social de las necesidades y combinando el derecho a satisfacer las necesidades con el derecho a participar en las decisiones de cómo alcanzar esa realización. 


\section{Referencias}

Barreto, Miguel.; Pelli, Víctor.; Alcalá, Laura.; Benítez, María.; Pelli, María; Giró, Marta.; Fernández, María E. y Romagnoli, Venettia (2010) "Análisis de Proyectos de mejoramiento del hábitat desde una perspectiva integral", en Peyloubet, P.; De Salvo, L. y Ortecho, E. (comps.) Hábitat. Fortalecimiento del espacio disciplinar en el sistema de Ciencia y Técnica. Buenos Aires, NOBUKO, 239-250.

Barreto, Miguel Á.; Alcalá, Laura; Benítez, María A.; Fernández, María E.; Giró, Marta; Pelli, María B. y Romagnoli, Venettia. Colab: Depetris, Noe (2014a) "La política Federal de Vivienda desde su implementación en el Gran Resistencia (20032007). Análisis y recomendaciones”. Buenos Aires: Diseño.

Barreto, Miguel Á.; Alcalá, Laura; Benítez, María A.; Fernández, María E.; Giró, Marta; Pelli, María B. y Romagnoli, Venettia. Colab: Depetris, Noe (2014b) "Análisis de la formulación e implementación del Programa Federal Solidaridad Habitacional en el barrio Chelliyí (Resistencia, Chaco)", en Barreto M. y Lentini M. (comps.) "Hacia una política integral de hábitat. Aportes para un observatorio de política habitacional en Argentina". Buenos Aires: Editorial Café de las ciudades, 417-492.

Barreto, Miguel Á.; Alcalá, Laura; Benítez, María A.; Fernández, María E.; Giró, Marta; Pelli, María B. y Romagnoli, Venettia. Colab: Depetris, Noel (2014c) "Un encuadre teórico-metodológico para el análisis de la política habitacional”, en Barreto M. y Lentini M. (comps.) "Hacia una política integral de hábitat. Aportes para un observatorio de política habitacional en Argentina”. Buenos Aires: Café de las ciudades, 143-212.

Bourdieu, Pierre (1993) "Esprits d'Etat”. Revista Actes de la Recherche No 96/97:49-62.

Bourdieu, Pierre (1998) La Distinción. Criterio y bases sociales del gusto, Madrid:Taurus Ediciones.

Bourdieu, Pierre (2001) Las estructuras sociales de la economía. Buenos Aires: Manantial.

Doyal, Len y Gough, Ian (1991). A Theory of Human Needs. London: Macmillan.

Doyal, Len y Gough, Ian (1994) Teoría de las necesidades humanas. Icaria, Barcelona.

Fraser, Nancy (1986) "Movimientos sociales vs. Burocracias disciplinarias: los discursos de las necesidades sociales” Diánoia, Vol. 32, n 32, 1986, p. 167-202.

Fraser, Nancy (1991) "La lucha por las necesidades: esbozo de una teoría crítica socialistafeminista de la cultura política del capitalismo tardío" Revista Debate Feminista, Marzo, 1991, p. 3-40.
Fraser, Nancy (1997a) Iustitia Interrupta: Reflexiones críticas desde la posición "postsocialista”, Santa Fe de Bogotá: Siglo del Hombre Editores, Universidad de los Andes.

Fraser, Nancy (1997b) “;De la redistribución al reconocimiento?. Dilemas en tomo a la justicia en una época "postsocialista". Disponible en: http://www.cholonautas.edu.pe modulo/upload/Fraser\%20cap1.pd] Fecha de consulta: 01/12/2012.

Fraser, Nancy (1999), "Repensando la esfera pública: una contribución a la crítica de la democracia actualmente existente", Revista Ecuador Debate No. 46, p. 139-174. Disponible en: http://repositorio.flacsoandes.edu.ec/bitstream/10469/5760/1/RFLACSO-ED4608-Fraser.pd). Fecha de consulta: 10/12/2012.

Gasper, Des (2002) "Is Sen's Capability Approach and Adequate Basis for Considering Human Development?" Review of Political Economy, Vol. 14, n 13, 2002, p. 435-461.

Gough, Ian (2003) Capital global, necesidades básicas y políticas sociales. Buenos Aires: Ed. Miño Davila-Ciepp.

Gough, Ian (2008) "Nussbaum: un análisis comparado con nuestra teoría de las necesidades humanas". Revista Papeles de Relaciones Ecosociales y Cambio Global, nº 100, CIPEcosocial/Icaria, invierno 2007/08.

Heller, Agnes (1986). Teoría de las necesidades en Marx. Barcelona: Península.

Heller, Agnes (1996). Una revisión de la teoría de las necesidades. Barcelona: Paidós e I.C.E de la Universidad Autónoma de Barcelona.

Jobert, Bruno (2005) Estado, Sociedad, Políticas Públicas. Santiago de Chile: LOM Editores.

Ibrahim, Solava S. (2006), "From Individual to Collective Capabilities:The Capability Approach as a Conceptual Framework for Self-help." Journal of Human Development, Vol 7 n 3, November 2006, p. 397-416.

Krmpotic, Claudia (1999) El concepto de necesidad y políticas de bienestar. Una lectura comparada de Heller, Sen y el GPID. Buenos Aires: Espacio editorial.

Maslow, Abraham (1954) Motivation and personality. New York: Harper.

Max Neef, Manfred; Elizalde, Antonio y Hopenhayn, Martín (1986) Desarrollo a escala humana. Chile: CEPAUR y Fundación Dag Hammarskjold. 
Nussbaum, Martha (1992) "Human functioning and social justice: in defense of Aristotelian essentialism”. Political Theory, Vol. 2, N 20, p. 202-246.

Pelli, Víctor S. (2006) Habitar, Participar, Pertenecer. Acceder a la vivienda, incluirse en la sociedad. Buenos Aires: Nobuko.

Romagnoli,Venettia (2011) Pautas para la optimización de Programas de Mejoramiento Barrial. Un estudio sobre sus fundamentos teóricos y experiencias en el AMGR (Chaco, Argentina). Berlín: Editorial Académica Española.

Romagnoli, Venettia (2012) "La configuración de las necesidades en las políticas habitacionales a partir del caso del AMGR (Chaco). Avances en la construcción de un enfoque teórico para su abordaje”, en Leoni, María S.; Leconte, Mariana y Sudar Klappenblach, Luciana (comp.) Libro de Actas. XXXII Encuentro de Geohistoria Regional. Resistencia: Instituto de Investigaciones Geohistóricas, CONICET- UNNE pp. 799 - 810.

Romagnoli, Venettia (2015a) :Qué vivienda necesitan los pobres”. Un estudio sobre la configuración de las necesidades en las políticas habitacionales vigentes a partir del caso del Área Metropolitana Gran Resistencia - AMGR (Chaco, Argentina), Tesis doctoral inédita. Doctorado en Ciencias Sociales de FLACSO.

Romagnoli, Venettia (2015b) "El proceso de construcción de las necesidades en las políticas habitacionales y su incidencia en la configuración del territorio a partir del caso del Barrio Chelilly de Resistencia (Chaco, Argentina)", en Carpio, María B.; Fantín, María A.; Leoni, María S. y Salinas, María L. (Comps.) Libro de Actas del XXXIV Encuentro de Geohistoria Regional. Resistencia Instituto de Investigaciones Geohistóricas, CONICET- UNNE. pp. 547-558.

Romagnoli, Venettia (2015b) "La disputa por la configuración de las necesidades habitacionales en las políticas públicas. Construcción de un marco interpretativo para su abordaje”, ADNea Arquitectura y Diseño del Nordeste Argentino, Vol. 3, No 3, p. 101-118

Sen, Amartya (1987) Commodities and captabilities. Oxford: Oxford UP.

(1992) Inequality reexamined. Nueva York: Russell Sage Fundation.

(1997) "Poor relative speaking", en: Resources, values and development, Massachusetts: Harvard UP, Cambridge. pp. 325-345.

(2000) Desarrollo y Libertad. México: Editorial Planeta.
Subirats, Joan (1989) Análisis de las políticas públicas y eficacia de la administración. Madrid: Instituto Nacional de Administración Pública.

Subirats, Joan (2001) “El análisis de las políticas públicas”. GAC SAINT No 15,Vol.3, p. 259-264.

Tamayo Sáez, Manuel (1997) “El análisis de las políticas públicas”, en: Bañón, Rafael y Carrillo, Ernesto (comp.), La nueva administración pública. Madrid:Alianza Universidad. pp. 2-22.

\section{Cómo citar este artículo:}

Romagnoli, Venettia (2017) "El proceso de construcción de las necesidades en las políticas habitacionales”. Revista Perspectivas de Políticas Públicas vol. 7 № 13: 